\title{
Influence of Cysteine Deprivation on Chlamydial Differentiation from Reproductive to Infective Life-cycle Forms
}

\author{
By I. ALLAN, ${ }^{1} \uparrow$ T. P. HATCH ${ }^{2}$ AND J. H. PEARCE ${ }^{1 *}$ \\ 1 Department of Microbiology, University of Birmingham, Birmingham B15 2TT, UK \\ 2 Department of Microbiology and Immunology, Center for the Health Sciences, University of \\ Tennessee, Memphis, Tennessee 38163, USA
}

(Received 1 May 1985; revised 19 August 1985)

\begin{abstract}
The effects of omission of individual amino acids from growth medium on the differentiation of Chlamydia trachomatis DK-20 (serotype E) during infection of cycloheximide-treated McCoy cells are described. As judged by inclusion body staining with acridine orange, omission of cysteine from the medium severely retarded differentiation of reproductive reticulate body $(\mathrm{RB})$ to infective elementary body (EB) forms. The effect appeared specific to cysteine in that omission of other amino acids had little or no effect on differentiation once RBs appeared. On restoration of cysteine, culture infectivity increased and inclusions contained organisms which, by cytochemical and morphological criteria, were differentiating to infective forms, indicating that cysteine deprivation did not irreversibly inhibit differentiation. Impairment of RB to EB differentiation in cysteine-less medium was also observed for three strains of Chlamydia psittaci and 10 other strains of $C$. trachomatis. It is suggested that the effect arises via the biosynthetic requirement for cysteine for provision of three cysteine-rich proteins, whose synthesis and insertion into the outer membrane have previously been shown to accompany RB to EB differentiation of $C$. psittaci $6 \mathrm{BC}$ and C. trachomatis 434 (serotype $\mathrm{L}_{2}$ ). Synthesis of cysteine-rich outer membrane proteins during differentiation may thus be common to all chlamydiae.
\end{abstract}

\section{INTRODUCTION}

Chlamydiae are obligate intracellular bacteria that undergo a unique developmental cycle within the host cell. Following endocytosis the small, rigid, infectious elementary body (EB) differentiates to the large, fragile, metabolically active reproductive or reticulate body (RB). After a period of binary fission, the latter redifferentiate to infectious forms and host cell lysis and release of progeny occurs.

The structural differences between RBs and EBs and their functional relationships with physiological properties of the two life-cycle forms are poorly understood. Nor is it known how the EB to RB to EB morphogenetic cycle is regulated. For transmissible infection the latter must ensure both division of reproductive forms and redifferentiation of these to infectious EBs.

Tamura \& Manire (1967), studying the CAL-10 strain of Chlamydia psittaci, reported that envelope preparations of RBs differed from those of EBs in that they contained neither cysteine nor methionine. Failing to detect muramic acid in chlamydial envelopes (Tamura \& Manire, 1967), they suggested that disulphide bonding might contribute to the structural rigidity of EBs that is lacking in the fragile RBs. Data supporting this proposal have been obtained by Hatch $e t$ al. (1981), who showed that solubilization of outer membrane proteins of the $6 \mathrm{BC}$ strain of $C$.

$\dagger$ Present address: Department of Paediatrics, John Radcliffe Hospital, University of Oxford, Headington, Oxford OX3 9DU, UK.

Abbreviations: EB, elementary body; RB, reticulate body; FBS, foetal bovine serum; HBSS, Hanks' balanced salts solution; i.f.u., inclusion-forming unit; p.i., post-infection. 
psittaci required both detergent and reducing agent. Further, Newhall \& Jones (1983) have reported the presence of disulphide cross-linked polypeptide complexes in outer membrane preparations of $C$. trachomatis UW94 (serotype E).

Stirling et al. (1983) presented evidence from metabolic studies that deprivation of cysteine during the growth of $C$. trachomatis DK-20 (serotype E) severely retarded differentiation of RB to EB forms. This led to the demonstration, for both $C$. psittaci $6 \mathrm{BC}$ and $C$. trachomatis 434 (serotype $\mathrm{L}_{2}$ ), that $\mathrm{RB}$ to $\mathrm{EB}$ differentiation is associated with the synthesis of three extraordinarily cysteine-rich outer membrane proteins which appear to be important for the structural rigidity of EBs (Hatch et al., 1984) and which may well influence, via surface structure, physiological properties unique to the infective EB. Here we have examined the specificity of amino acid deprivation on differentiation of strain DK-20 and assessed the effect of cysteine deprivation on differentiation of 13 other chlamydial strains.

\section{METHODS}

Organisms. Chlamydiae were produced by growth in irradiated McCoy cell monolayers; inoculation, incubation and preparation were as previously described (Allan \& Pearce, 1983b). The following strains were grown. $C$. trachomatis strains A/SA-1/OT (Wang \& Grayston, 1971), B/TW-5/OT, C/UW-1/OT, D/CAL-8/ON (Treharne et al., 1977), E/MRC-4/IC (Collier \& Smith, 1967), E/DK-20/ON, F/MRC-301/U, G/IOL-238/R, H/UW-4/Cx, $\mathrm{I} / \mathrm{UW}-12 / \mathrm{U}$ and $\mathrm{L}_{2} / 434 / \mathrm{Bu}$ (Treharne et al., 1977): the classification is serotype/strain/origin; OT, ocular trachoma; ON, ophthalmia neonatorum; IC, inclusion conjunctivitis; $\mathrm{U}$, urethra; $\mathrm{R}$, rectum; $\mathrm{Cx}$, cervix; $\mathrm{Bu}$, bubo. C. psittaci strains enzootic abortion of ewes (EAE; Stamp et al., 1950), guinea pig inclusion conjunctivitis (GP-IC; Murray, 1964), and meningopneumonitis (CAL-10; Francis \& Magill, 1938).

Cell culture and monolayer preparation. These were as previously described for irradiated McCoy cells (Allan \& Pearce, 1983b).

Assessment of the effect on chlamydial development of deletion of amino acids from the growth medium. Suspensions of chlamydiae in Hanks" balanced salts solution (HBSS) containing $5 \%(\mathrm{v} / \mathrm{v})$ foetal bovine serum (FBS) were centrifuged with monolayers of McCoy cells irradiated one day earlier (Griffiths et al., 1976). Subsequently the inoculum was removed and control or test growth medium added. Growth media were prepared from a "select amine kit' (Gibco Biocult, Paisley, UK) as previously described (Allan \& Pearce, 1983a) and were supplemented with streptomycin $\left(100 \mu \mathrm{g} \mathrm{ml}^{-1}\right)$ and cycloheximide $\left(2 \mu \mathrm{g} \mathrm{ml}^{-1}\right)$; FBS was dialysed before supplementation to $5 \%$ (v/v). Monolayers were incubated at $35^{\circ} \mathrm{C}$ in air/ $\mathrm{CO}_{2}(95: 5, \mathrm{v} / \mathrm{v})$ and sampled at intervals for assessment of one or more of the parameters considered below.

Numbers of infected cells. These were counted by microscopic examination of infected monolayers after fixation and Giemsa staining

Yield of infectious progeny. This was determined as previously described (Allan \& Pearce, 1983a).

Cytochemical composition of deteloping inclusions. This was assessed by microscopic examination of infected monolayers after fixation with cold glacial acetic acid:ethanol $(1: 2, \mathrm{v} / \mathrm{v})$ and staining with acridine orange fluorescence (Pollard \& Tanami, 1962). Infected monolayers were scanned (500 $\times$ magnification) using an oil immersion planapochromat objective $(40 \times$; Carl Zeiss) for the presence of inclusions containing red RB and limegreen EB forms; these had been verified by electron microscopic examination of infected monolayers (Stirling $e t$ al., 1983).

In all experiments with $C$. trachomatis DK-20, except where stated, one cytochemical form appeared to predominate, both in individual inclusions and in infected cell populations. In a few instances (see text) inclusions contained significant numbers of forms intermediate in fluorescence between RBs and EBs, although appearing EB-like in size; these were recorded as intermediate forms. In two experiments a range of strains of $C$. trachomatis and $C$. psittaci were examined (Table 3). Here, although individual inclusions contained largely one cytochemical form, variation between infected cells was apparent. Inclusions contained largely either RBs or EBs and were subjectively graded $0,1,2,3$ or 4 , corresponding, approximately to $0,25,50,75$ or $100 \%$ of inclusions scanned being of one or other form. Thus, monolayers graded ' 2,2 ' contained approximately equal numbers of inclusions in predominantly RB or EB form; monolayers graded ' 3 , 1 ' or ' 1,3 ' contained $75 \%$ and $25 \%$, respectively, of RBdominant inclusions.

\section{RESULTS}

\section{Specificity of effect of amino acid deprivation on development of life-cycle forms of C. trachomatis $D K-20$}

Deprivation of cysteine from growth medium markedly delayed the differentiation of RB to EB forms within inclusions in cells infected with C. trachomatis (oculogenital strain DK-20, 
serotype E) as judged by both acridine orange staining and electron microscopy (Stirling et al., 1983). This might have arisen as a result of a general inhibition of inclusion body formation, as seen in earlier studies following deprivation of glutamine, histidine, leucine, phenylalanine or valine (Allan \& Pearce, $1983 \mathrm{~b}$ ). These experiments with strain DK-20 were therefore repeated with acridine orange staining so that the $\mathrm{RB}$ and $\mathrm{EB}$ content of inclusions, and thus possible interference with differentiation, could be assessed (Table 1). Observation was extended to $72 \mathrm{~h}$ post-infection (p.i), which, for the irradiated treated cell monolayers under study, approached the maximum time over which infected fully-supplemented cultures could be examined unobscured by cell deterioration.

Of those amino acids whose omission did not impair infection only absence of cysteine markedly retarded RB to EB development (Table 1). Deprivation of leucine, which inhibited infection by G, H, I but not D, E, F serotypes of oculogenital strains (Allan \& Pearce, 1983b), delayed appearance of inclusions to $48 \mathrm{~h}$ p.i.; at this time these were RB in content but by $72 \mathrm{~h}$ were entirely EB.

For amino acids whose omission inhibited infection, and which defined nutritional biotypes of $C$. trachomatis (Allan \& Pearce, 1983 b), the effect of deprivation upon development was similar to the above. Thus, for glutamine and phenylalanine, inclusion formation was absent at $24 \mathrm{~h}$ p.i., small RB-containing inclusions were evident at $48 \mathrm{~h}$ and these had converted to EB by $72 \mathrm{~h}$. Deprivation of histidine or valine inhibited appearance of inclusions until $72 \mathrm{~h}$ p.i. For valine, the latter were RB-containing but further incubation to test for differentiation to EB was not feasible.

\section{Effect of cysteine deprivation and restoration on differentiation of C. trachomatis DK-20 to infectious life-cycle forms}

One explanation for the retardation of RB to EB differentiation during cysteine deprivation was that structurally or metabolically abnormal forms were induced which were incapable of differentiation to EB forms. To test this possibility the effect of restoration of cysteine on chlamydial differentiation and appearance of infectivity was examined, following a period of deprivation.

Inclusions in infected monolayers $48 \mathrm{~h}$ p.i. and under cysteine deprivation contained RB forms, but with a low background of infectivity which had declined by $72 \mathrm{~h} \mathrm{p}$.i. (Table 2). Restoration of cysteine for $24 \mathrm{~h}$ led to a significant increase in infectivity over that in control infected monolayers still deficient in cysteine or to which cysteine and chloramphenicol (for inhibition of chlamydial protein synthesis) had been added (Table 2). It thus appeared that chlamydiae present after deprivation of cysteine for $48 \mathrm{~h}$ were capable of differentiation to infective forms, at least to some extent, on addition of cysteine.

\section{Effect of amino acid deprivation on the development of life-cycle forms of C. trachomatis and C. psittaci}

Having established the specificity of cysteine deprivation on RB to EB differentiation for strain DK-20, it was important to assess the general validity of the effect. In addition, the earlier work of Tamura \& Manire (1967) had suggested that differences in the content of both methionine and cysteine existed between the envelopes of RB and EB forms of C. psittaci CAL10. We therefore compared cysteine, methionine and combined cysteine and methionine deprivation on the developmental cycle of a range of strains of $C$. trachomatis and C. psittaci. Since from earlier data deprivation of either arginine, isoleucine, lysine, threonine, tryptophan or tyrosine had not impaired infection by oculogenital strains of $C$. trachomatis and certain strains of C. psittaci (Allan \& Pearce, 1983b), the effect of en bloc omission of these was also examined.

For all strains examined, deprivation of cysteine retarded differentiation of RBs to EBs (Table 3); in no case, however, was this enhanced by the combined deprivation of cysteine and methionine. Methionine omission alone did not retard, except marginally for strain CAL-10, at $24 \mathrm{~h}$ p.i. Absence of six other amino acids as a group was also without effect. Overall, it appeared 
Table 1. Effect of single amino acid omissions on the infectivity and development of life-cycle forms of $C$. trachomatis $D K-20$ in cycloheximide-treated monolayers

Suspensions of strain DK-20 in HBSS supplemented with 5\% FBS were centrifuged with irradiated McCoy cell monolayers and, after removal of inoculum, growth medium containing 12 of the 13 amino acids of Eagle's minimal essential medium, $5 \%$ dialysed FBS and $2 \mu \mathrm{g}$ cycloheximide $\mathrm{ml}^{-1}$ was added. Monolayers $48 \mathrm{~h}$ p.i. were fixed and stained with Giemsa for counts of infected cell numbers and monolayers treated in parallel ( 24 and 48 and $72 \mathrm{~h}$ p.i.) were fixed and stained with acridine orange for assessment of the cytochemical composition of inclusions. In control monolayers, which were incubated with complete growth medium, approx. $95 \%$ of cells were infected. The results are for one experiment; individual values are the mean of duplicate monolayers with approx. 800 cells per monolayer scanned after Giemsa staining and 1000 cells after acridine orange staining.

\begin{tabular}{|c|c|c|c|c|}
\hline \multirow{2}{*}{$\begin{array}{l}\text { Omitted } \\
\text { amino acid }\end{array}$} & \multirow[b]{2}{*}{ Infection* } & \multicolumn{3}{|c|}{$\begin{array}{l}\text { Dominant cytochemical form } \\
\text { within inclusion bodies }\end{array}$} \\
\hline & & 24 h p.i. & 48 h p.i. & 72 h p.i. \\
\hline$\ldots$ & 100 & RB & EB & EB \\
\hline Arginine & 96 & RB & $\mathrm{RB}>\mathrm{IF}+$ & EB \\
\hline Cysteine & 95 & RB & RB & RB \\
\hline Glutamine & 18 & 0 & $\mathrm{RB} \ddagger$ & $\mathrm{EB} \ddagger$ \\
\hline Histidine & 0 & 0 & 0 & $\mathrm{~EB}+\S$ \\
\hline Isoleucine & 101 & $\mathrm{RB}$ & $\mathrm{EB}>\mathrm{IF} \dagger$ & EB \\
\hline Leucine & 97 & 0 & RB $\ddagger$ & EB \\
\hline Lysine & 97 & RB & IFt & EB \\
\hline Methionine & 96 & $\mathrm{RB}$ & EB & EB \\
\hline Phenylalanine & 4 & 0 & RB $\ddagger$ & EB \\
\hline Threonine & 96 & $\mathrm{RB}$ & IFt & EB \\
\hline Tryptophan & 98 & $\mathrm{RB}$ & $\mathrm{EB}>\mathrm{IF} \dagger$ & EB \\
\hline Tyrosine & 95 & $\mathrm{RB}$ & $\mathrm{EB}>\mathrm{IF}+$ & $\mathrm{EB}$ \\
\hline Valine & 0 & 0 & 0 & RB $\$$ \& \\
\hline
\end{tabular}

* Infection is expressed as a percentage of the number of infected cells in the control receiving complete medium.

$\dagger$ Organisms appearing intermediate in fluorescence colour between typical RBs and EBs, although EB-like in size, were recorded as IF (intermediate forms). In some instances inclusions contained both RB and IF or IF and EB forms.

$\ddagger$ Inclusions were markedly smaller than those in cells in complete medium.

$\S$ Less than $1 \%$ of cells in the monolayer contained inclusions.

\section{Table 2. Effect of restoration of cysteine on the differentiation of C. trachomatis DK-20 to infective forms in cell monolayers}

Infected monolayers (approx. 95\% cells infected) were incubated for $48 \mathrm{~h}$ p.i. in medium with cysteine (Cys) present or absent. Cysteine-less cultures were denied cysteine for a further $24 \mathrm{~h}$ or replenished with cysteine, with or without the addition of chloramphenicol $\left(80 \mu \mathrm{g} \mathrm{ml}^{-1}\right)$. Infected monolayers were assessed at these times for the cytochemical composition of inclusions in infected cells and, in parallel monolayers, for their yield of infective progeny. Results are the mean of two experiments; estimates of cytochemical composition are from examination of duplicate monolayers, 1500 cells per monolayer; infectivity yields were titrated from four monolayers pooled after sonication and inclusion-forming units (i.f.u.) per sonicated cell were calculated from mean inclusion counts from duplicate monolayers after scanning 2500 cells per monolayer (approx. $10 \%$ cells infected).

\begin{tabular}{|c|c|c|c|}
\hline $0-48 \mathrm{~h}$ & $\overline{48-72 \mathrm{~h}}$ & $\begin{array}{l}\text { Dominant cytochemical } \\
\text { form in infected cells }\end{array}$ & $\begin{array}{c}\text { Yield } \\
\text { (i.f.u. per infected cell) }\end{array}$ \\
\hline + Cys & - & EB & 590 \\
\hline - Cys & - & RB & $17 \cdot 5$ \\
\hline -Cys & - Cys & RB & 0.2 \\
\hline - Cys & $+\mathrm{Cys}+\mathrm{Cpl}$ & $\mathrm{RB}$ & $0^{*}$ \\
\hline - Cys & + Cys & $\mathrm{IF} \dagger$ & $7 \cdot 1$ \\
\hline
\end{tabular}

* Failure to detect infectivity in this sample may be due to contaminating chloramphenicol (Cpl). † Intermediate forms (see Table 1). 
Table 3. Effect of omission of sulphur-containing or 'non-required' amino acids on the development of strains of $C$. trachomatis and $C$. psittaci in cell monolayers

Infection conditions were as described in Table 1 except that media were deficient either in cysteine, methionine, cysteine and methionine or a "non-required" group of six amino acids comprising arginine, isoleucine, lysine, threonine, tryptophan and tyrosine. At the times indicated p.i. monolayers were fixed and stained with acridine orange for assessment of cytochemical composition of inclusions. Approx. 1000 cells on each of two monolayers were scanned; individual inclusions were predominantly RB or EB and the proportion in one or other form was graded $0,1,2,3$ or 4 corresponding to 0 to $100 \%$ (see Methods). In monolayers prepared in parallel, strain infectivity in complete medium was assessed by staining of monolayers ( 800 cells scanned on each of duplicate monolayers) 48 h p.i. (C. trachomatis) or $24 \mathrm{~h} \mathrm{p.i.} \mathrm{(C.psittaci)} \mathrm{and} \mathrm{compared} \mathrm{with} \mathrm{the} \mathrm{values} \mathrm{for} \mathrm{deficient} \mathrm{media.} \mathrm{Results} \mathrm{are} \mathrm{for} \mathrm{one} \mathrm{experiment}$ (cysteine deprivation data are typical of results for two experiments); between 60 and $95 \%$ of cells were infected by the strain inocula tested.

Strain Omitted amino acids

Proportion of inclusions in RB- or EB-dominant form

\begin{tabular}{|c|c|c|c|}
\hline Strain & Omitted amino acids & $\mathrm{RB}, \mathrm{EB}$ & RB, EB \\
\hline \multicolumn{4}{|c|}{ C. trachomatis (serotype) } \\
\hline \multirow{5}{*}{ CAL-8(D) } & Cys, Met & 2,2 & 2,2 \\
\hline & Cys & 2,2 & 2,2 \\
\hline & Met & 0,4 & 0,4 \\
\hline & Arg, Ile, Lys, Thr, Try, Tyr & 0,4 & 0,4 \\
\hline & None & 0,4 & 0,4 \\
\hline \multirow[t]{3}{*}{$\mathrm{MRC}-4(\mathrm{E})^{*}$} & Cys, Met & 2,2 & 0,4 \\
\hline & Cys & 2,2 & 0,4 \\
\hline & Met & 0,4 & 0,4 \\
\hline \multirow[t]{3}{*}{ DK $-20(E)^{*}$} & Cys, Met & 4,0 & 3,1 \\
\hline & Cys & 4,0 & 3,1 \\
\hline & Met & 0,4 & 0,4 \\
\hline \multirow{3}{*}{$\mathrm{MRC}-301(\mathrm{~F})^{*}$} & Cys, Met & 2,2 & 2,2 \\
\hline & Cys & 2,2 & 2,2 \\
\hline & Met & 0,4 & 0,4 \\
\hline \multirow[t]{3}{*}{ IOL-238(G)* } & Cys, Met & 2,2 & 1,3 \\
\hline & Cys & 2,2 & 1,3 \\
\hline & Met & 0,4 & 0,4 \\
\hline \multirow[t]{3}{*}{ UW-4(H)* } & Cys, Met & 4,0 & 1,3 \\
\hline & Cys & 4,0 & 1,3 \\
\hline & Met & 0,4 & 0,4 \\
\hline \multirow[t]{3}{*}{ UW-12(I)* } & Cys, Met & 4,0 & 2,2 \\
\hline & Cys & 4,0 & 2,2 \\
\hline & Met & 0,4 & 0,4 \\
\hline $\mathrm{SA}-1(\mathrm{~A}) \dagger$ & Cys & 3,1 & ND \\
\hline TW-5(B)† & Cys & 3,1 & ND \\
\hline UW-1(C)† & Cys & 3,1 & ND \\
\hline \multirow[t]{2}{*}{$434\left(\mathrm{~L}_{2}\right) \dagger$} & Cys & 2,2 & ND \\
\hline & & 24 h p.i. & $48 \mathrm{~h}$ p.i. \\
\hline \multicolumn{2}{|l|}{ C. psittaci } & RB, EB & $\mathrm{RB}, \mathrm{EB}$ \\
\hline \multirow[t]{3}{*}{ GP-IC $*$} & Cys, Met & 4,0 & 1,3 \\
\hline & Cys & 4,0 & 1,3 \\
\hline & Met & 2,2 & 0,4 \\
\hline \multirow[t]{3}{*}{ CAL-10* } & Cys, Met & 2,2 & 1,3 \\
\hline & Cys & 3,1 & 1,3 \\
\hline & Met & 2,2 & 0,4 \\
\hline EAE $†$ & Cys & 4,0 & ND \\
\hline
\end{tabular}

ND, Not determined.

* Results for complete medium or omission of the 'non-required' group of amino acids were identical to those for omission of methionine. Omissions did not reduce infectivity compared with that in complete medium.

$\dagger$ In complete medium all inclusions were $\mathrm{EB}$ dominant $(\mathrm{RB}, \mathrm{EB}=0,4)$ at $48 \mathrm{~h} \mathrm{p.i.}(\mathrm{C}$. trachomatis $)$ or contained equal numbers of $\mathrm{RB}$ and $\mathrm{EB}(\mathrm{RB}, \mathrm{EB}=2,2)$ at $24 \mathrm{~h}$ p.i. (C. psittaci $\mathrm{EAE})$; omissions did not reduce infectivity compared with that in complete medium. 
that only cysteine deprivation significantly affected differentiation. Interestingly, the extent of retardation appeared to vary between strains, with DK-20, the strain first examined for these effects, apparently the most affected.

\section{DISCUSSION}

For strain DK-20, omission of cysteine from the medium severely retarded RB to EB differentiation without impairment of infectivity (Table 1). Deletion of leucine, glutamine, histidine, phenylalanine or valine (Table 1) reduced inclusion size and/or infected cell number and thus substantially interfered with growth; nevertheless, with the possible exception of valine, conversion of RB to EB forms was not impaired. We conclude, therefore, that retardation of differentiation is specific to deprivation of cysteine and does not occur as a result of generalized slowing of chlamydial protein synthesis and RB division.

Only a moderate rise in infectivity occurred when cysteine-deprived monolayers were replenished with cysteine (Table 2). It is possible that absence of cysteine led to the formation of a proportion of morphologically and metabolically abnormal RBs. However, the presence in such inclusions $72 \mathrm{~h}$ p.i. of what were predominantly intermediate forms suggests that the low infectivity appearing subsequent to cysteine restoration was due to uncompleted differentiation of the majority of organisms present rather than to failure of the majority to differentiate from a state of irreversible inhibition. As in the earlier experiment with valine deprivation (Table 1), it was not possible to extend the period of examination because of monolayer deterioration. The latter, via inactivation, may have contributed to the apparent decline in recoverable infectivity over 48 to $72 \mathrm{~h}(17.5$ versus 0.2 ; Table 2$)$. Inhibition by chloramphenicol of the appearance of infectivity, despite the presence of cysteine, provides further evidence that a true increase had occurred on cysteine restoration, emphasizing that $\mathrm{RB}$ to $\mathrm{EB}$ differentiation requires protein synthesis in the presence of cysteine and does not occur merely by reorganization from existing RB components.

Extension of cysteine deprivation studies to other strains of $C$. trachomatis and $C$. psittaci indicated that differentiation was retarded for all 13 strains examined (Table 3). This was significant in each case compared with strain behaviour in complete medium or in medium lacking the six amino acids arginine, isoleucine, lysine, threonine, tryptophan and tyrosine. It is of interest that, in a previous analysis of $C$. psittaci GP-IC (Allan \& Pearce, 1983 b), only a marginal reduction of infectivity occurred on cysteine deprivation for inclusions harvested at $40 \mathrm{~h}$ p.i., which presumably retained similar perceptible numbers of RBs as observed here at $48 \mathrm{~h}(\mathrm{RB}, \mathrm{EB}=1,3$; Table 3).

We have shown elsewhere that for $C$. psittaci 6BC and $C$. trachomatis LGV $434 \mathrm{RB}$ to EB differentiation is accompanied by the synthesis and appearance in outer membrane complexes of three cysteine-rich proteins (Hatch et al., 1984), one or more of which may be similar to the keratin-like spore coat proteins in cysteine content (Aronson \& Fitz-James, 1968). It is possible therefore that there is a considerable biosynthetic demand for cysteine during differentiation and this may account for the retardation which we have now shown to be cysteine-specific. Supportive evidence from estimates of molar ratios and cysteine contents of envelope proteins of $\mathrm{RB}$ and EB forms is not yet available. It is, however, clear from comparative radiolabelling experiments that the cysteine-rich proteins contain cysteine in amounts very significantly above either that in total RB proteins or the major outer membrane protein, which contains both cysteine and methionine and is common to both RB and EB envelopes (Hatch et al., 1984).

Although cysteine deprivation, following general nutrient starvation, can inhibit inclusion formation (Karayiannis \& Hobson, 1981), omission here did not appear to prevent RB division which entails incorporation of cysteine into RB proteins, Thus, if the biosynthetic demand hypothesis is correct, it is the fulfilment of cysteine-rich protein synthesis during differentiation, rather than total protein synthesis, which is impaired by cysteine deprivation. In one other instance where the cysteine level influences differentiation the effect on metabolism is more general: transition of Histoplasma capsulatum from the mycelial to the yeast form requires cysteine to reactivate mitochondrial metabolism via shunt pathways which bypass components of the cytochrome system lost during the transition (Sacco et al., 1983). 
As discussed elsewhere, the failure of Tamura \& Manire (1967) to detect cysteine and methionine in $\mathrm{RB}$ outer membrane preparations may have resulted from exposure of $\mathrm{RBs}$ to $0.5 \%$ SDS which, for RBs of strains $6 \mathrm{BC}$ and LGV 434 , extracted a large proportion of envelope proteins (Hatch et al.,1984). The absence of significant retardation of differentiation following methionine deprivation (Table 3 ) is in accord with our observations on $\left[{ }^{35} \mathbf{S}\right]$ methionine and ${ }^{[35}$ S]cysteine labelling of polypeptides in strains $6 \mathrm{BC}$ and LGV 434 . These showed that the major outer membrane protein is the major methionine-containing protein and that it is present in both RB and EB envelopes, probably in quantitatively similar amounts (Hatch et al., 1984).

The fact that all 14 strains were retarded during cysteine deprivation suggests that the strategy of biosynthesis of cysteine-rich proteins during RB to EB differentiation is common to all chlamydiae. It is of interest that DK-20, the C. trachomatis strain first examined, was the one most markedly impaired (Table 3 ). It is possible that such variations could reflect significant differences of detail, between chlamydiae, in envelope content of cysteine-rich proteins and deployment of disulphide bond cross-linking.

We thank the Medical Research Council for financial support.

\section{REFERENCES}

Allan, I. \& Pearce, J. H. (1983a). Differential amino acid utilization by Chlamydia psittaci (strain guinea pig inclusion conjunctivitis) and its regulatory effect on chlamydial growth. Journal of General Microbiology 129, 1991-2000.

Allan, I. \& Pearce, J. H. (1983b). Amino acid requirements of strains of Chlamydia trachomatis and C. psittaci growing in McCoy cells: relationship with clinical syndrome and host origin. Journal of General Microbiology 129, 2001-2007.

Aronson, A. I. \& Fitz-James, P. C. (1968). Biosynthesis of bacterial spore coats. Journal of Molecular Biology 33, 199-212.

Collier, L. H. \& SMITH, A. (1967). Dissemination and immunogenicity of live TRIC agent in baboons after parenteral injection. American Journal of Ophthalmo$\log y 63,1589-1602$.

Francis, T. \& Magill, T. P. (1938). An unidentified virus producing acute meningitis and pneumonitis in experimental animals. Journal of Experimental Medicine 68, 147-160.

Griffiths, M. S., Ainsworth, S. \& Pearce, J. H. (1976). Infectivity titration of guinea-pig inclusion conjunctivitis agent in irradiated McCoy cells. Journal of General Microbiology 95, 249-256.

Hatch, T. P, Vance, D. W., JR \& Al-Hossainy, E. (1981). Identification of a major envelope protein in Chlamydia spp. Journal of Bacteriology 146, 426429.

Hatch, T. P., Allan, I. \& Pearce, J. H. (1984). Structural and polypeptide differences between envelopes of infective and reproductive life cycle forms of Chlamydia spp. Journal of Bacteriology 157, 13-20.

KaRayiannis, P. \& Hobson, D. (1981). Amino acid requirements of a Chlamydia trachomatis genital strain in McCoy cell cultures. Journal of Clinical Microbiology 13, 427-432.

MurRay, E. S. (1964). Guinea pig inclusion conjunctivitis virus. I. Isolation and identification as a member of the psittacosis-lymphogranuloma- trachoma group. Journal of Infectious Diseases 114, $1-12$.

Newhall, W. J. \& Jones, R. B. (1983). Disulfidelinked oligomers of the major outer membrane protein of chlamydiae. Journal of Bacteriology 154, 998-1001

Pollard, M. \& Tanami, Y. (1962). Cytochemistry of trachoma virus replication in tissue cultures. Annals of the New York Academy of Sciences 98, 50-61.

SaCco, M., Medoff, G., Lambowitz, A. M., Kumar, B. V., Kobayashi, G. S. \& Painter, A. (1983). Sulfhydryl induced respiratory 'shunt' pathways and their role in morphogenesis in the fungus, Histoplasma capsulatum. Journal of Biological Chemistry 258, 8223-8230.

Stamp, J. T., McEwen, A. D., Watt, J. A. A. \& Nisbet, D. J. (1950). Enzootic abortion in ewes. I. Transmission of the disease. Veterinary Record 62, 251-254.

Stirling, P., Allan, I. \& Pearce, J. H. (1983). Interference with transformation of chlamydiae from reproductive to infective body forms by deprivation of cysteine. FEMS Microbiology Letters 19, $133-136$.

Tamura, A. \& Manire, G. P. (1967). Preparation and chemical composition of the cell membranes of developmental reticulate forms of meningopneumonitis organisms. Journal of Bacteriology 94, 11841188.

Treharne, J. D., Dines, R. J. \& Darougar, S. (1977). Serological responses to chlamydial ocular and genital infections in the United Kingdom and Middle East. In Nongonococcal Urethritis and Related Infections, pp. 249-258. Edited by K. K. Holmes \& D. Hobson. Washington, DC: American Society for Microbiology.

Wang, S. P. \& Grayston, J. T. (1971). Classification of TRIC and related strains with micro-immunofluorescence. In Trachoma and Related Disorders, pp. 305-321. Edited by R. L. Nichols. Amsterdam: Excerpta Medica. 